Methods Blood samples collected from consenting 133 males and 305 female participants in Ibadan were tested for HBsAg, HBeAg, HBcIgM, HBcTotal and HBsAb by ELISA technique. Samples positive for HBsAg were further analysed for $\mathrm{HBV}$ DNA by amplifying and sequencing the $S$ gene. Isolates were genotyped and subtyped based on amino acid residues at position $122,127,134,160$ of the $S$ gene.

Results Of the 438 subjects tested 31 (7.1\%) were positive for HBsAg, 2 (6.5\%) of which were $\mathrm{HBeAg}$ positive. Ninety-nine (22.8\%) had detectable HBsAb, $3(0.7 \%)$ were positive for HBcIgM and 195 (44.5\%) were HBcTotal positive. HBV DNA was amplified and sequenced in 27 out of 31 and 4 could not be amplified due to low titres. After sequencing, 9 (33.3\%) were not exploitable due to the presence of multiple peaks. Of the 18 exploitable isolates, only 15 showed significant similarity to HBV S-gene. Eleven of the 15 isolates were subtyped as ayw4 while others could not due to substitution at $s 122 \mathrm{p}$. Phylogram showed that the 11 isolates were genotype E. Two of the 4 isolates with $\mathrm{R} 122 \mathrm{Q} / \mathrm{P}$ substitutions also belonged to genotype $\mathrm{E}$ while the other 2 which were $>11 \%$ divergent from the reference genotype E sequence clustered with an isolate previously described as an Immune Escape Mutant.

Conclusion This study identified high endemicity of HBV infection, presence of markers of infection even in non-detectable HBsAg levels and circulation of genotype $\mathrm{E}$ ayw4 and vaccine mutants in south-western Nigeria. It therefore emphasises the risk of development of an indigenous infected population that may not be protected by the current vaccine.

\section{PO 8276 COMMUNITY INDEX CASE APPROACH AND HIV TESTING AND COUNSELING (HTC) FOR SEXUAL PARTNERS OF HIV-POSITIVE PATIENTS LOST TO FOLLOW-UP: THE EXPERIENCE OF WORLD VISION MOZAMBIQUE}

Miguelhete Lisboa*, Saraiva Culuze, Saimado Imputiua, Adolfo Cambule, Christine Latif. World Vision Mozambique, Mozambique

\subsection{6/bmjgh-2019-EDC.66}

Background HIV-positive patients lost to follow-up (HP-LTFU) represent a challenge for HIV/AIDS control efforts as they are associated with higher risk of HIV transmission to their sexual partners, low viral load suppression and higher risk of morbidity and mortality than adherent patients. The SCIP-Ogumaniha programme implemented by World Vision Mozambique, has been utilising the index case approach together with systematic home-based HIV testing and counseling (hHTC) since August 2016 in 7 districts of the Zambezia province. This abstract outlines an evaluation of the contribution of this approach to HIV/AIDS care and treatment (HACT) of sexual partners of HP-LTFU in alignment with the first and second targets of the 90-90-90 UNAIDS strategy.

Methods The study involved HP-LTFU returned to HACT between October 2016 and September 2017. These patients reported to have sexual partners who had not been tested for HIV and provided informed, written consent for joint hHTC with these individuals. The hHTC package for sexual partners was offered by World Vision project counselors and those who tested HIV-positive were referred to HACT.

Results Of 7.084 patients who returned to HACT and reported to have an untested sexual partner, 63\% $(4,471)$ provided informed, written consent for joint hHTC. Of 4264 sexual partners found and tested, 52\% was female, $64 \%$ was in the 15-34 age groups, and 88\% had never been tested for HIV. About 28\% (1.205/4.264) was HIV-positive, 56\% of the sexual partners who tested HIV-positive, was female and $98 \%$ of these was successfully referred to HACT.

Conclusion The index case approach together with hHTC has contributed to the early diagnosis of $28 \%$ of new HIV infections among sexual partners of HP-LTFU and $98 \%$ of them ensured timely linkage to the HACT. Therefore, broader promotion and adoption of this approach would make a significant contribution to achievement of the first and second targets of the 90-90-90 UNAIDS strategy.

\section{PO 8278 BUILDING CAPACITY IN CONDUCTING CLINICAL RESEARCH IN A VIRTUAL SETTING: EXPERIENCES FROM THE EAST AFRICAN CONSORTIUM FOR CLINICAL RESEARCH (EACCR2)}

${ }^{1}$ Emily Nyanzi-Kabuye*, ${ }^{1}$ Pontiano Kaleebu, ${ }^{1}$ Benard Kikaire, ${ }^{2}$ Blandina T Mmbaga, ${ }^{3}$ Godfrey S Mfinanga, ${ }^{4}$ Norbert Peshu, ${ }^{5}$ Maowia Mukhtar. ${ }^{1}$ Uganda Virus Research Institute, Entebbe, Uganda; ${ }^{2}$ Kilimanjaro Clinical Research Institute, Tanzania; ${ }^{3}$ National Institute of Medical Research -Muhimbili, Tanzania; ${ }^{4}$ Kenya Medical Research Institute- Wellcome Trust, Nairobi, Kenya; ${ }^{5}$ nstitute of Endemic Diseases, University of Khartoum, Sudan

\subsection{6/bmjgh-2019-EDC.67}

Background EACCR2 is an EDCTP-funded, Eastern Africanled network established in May 2009, with 23 regional partners from Ehtiopia, Kenya, Sudan, Tanzania, and Uganda, and 8 northern partners from Germany, Netherlands, Norway, Sweden and United Kingdom. The objective is to strengthen capacity to conduct health research to international standards with specific focus on clinical trials on poverty-relevant diseases such as HIV, TB, malaria and neglected infectious diseases. EACCR2 optimises the use of shared research infrastructures and other regional capacity building resources and opportunities.

Activities The activities of the network are implemented in five work packages cutting across 'nodes' in different countries. Capacity building programmes and studies funded by EDCTP are implemented by coordinators at the disease nodes. The Uganda Virus Research Institute (UVRI) hosts the secretariat of the consortium of five nodes located in the following institutions: Malaria Node in Kilifi-Kenya Medical Research Institute Wellcome Trust, Tanzania; Training Node in Kilimanjaro Clinical Research Institute, Tanzania; Tuberculosis Node at the National Institute of Medical Research- Muhimbili, Tanzania; the Neglected and Re-Emerging Tropical Diseases Node at the University of Khartoum, Sudan; and the HIV Node at UVRI, Uganda.

Coordinators form the project implementation committee which meets via skype or teleconference every quarter to assess progress, share best practice and challenges of the network. Scientific and annual meetings are arranged every year in one of the implementing institutions. During such meetings, students, the nodes and steering committee also meet to minimise travel costs while helping teams to network.

EACCR2 learns from the experiences, best practice and challenges of EACCR1 while implementing its current activities. Careful planning and consensus building from all partners has been the driving force to build and implement activities of this virtual network. EACCR2 also works closely with other EDCTP Networks of Excellence, i.e. in Central 
Africa (CANTAM), West Africa (WANETAM), and Southern Africa (TESA).

\section{PO 8289 ASSOCIATION BETWEEN PLASMA LEVELS OF IL-27, IL-6 CYTOKINES AND P. FALCIPARUM INFECTION IN PREGNANT WOMEN LIVING IN MBALMAYO, CAMEROON}

1,2Balotin Fogang* ${ }^{*}$ ' Jean C Djontu, ${ }^{1}$ Rosette Megnekou, ${ }^{2}$ Lawrence Ayong. ${ }^{1}$ University of Yaounde I, Yaounde, Cameroon; ${ }^{2}$ Centre Pasteur du Cameroon, Yaounde, Cameroon

\subsection{6/bmjgh-2019-EDC.68}

Background The appropriate balance between anti-inflammatory and pro-inflammatory cytokines is necessary for protection against pregnancy-associated malaria and poor pregnancy outcomes. This study therefore aims to investigate the relationship between plasma levels of some regulatory cytokines and $P$. falciparum infection in Cameroonian women during pregnancy.

Methods Peripheral blood was collected from 131 women during pregnancy and 27 non-pregnant women living in the Mbalmayo area between May and December 2014. Parasitaemia was determined by microscopy and haemoglobin level using a haematological counter. Plasma levels of IL-27 and IL6 cytokines were measured using the Magnetic Luminex Screening Assay technique.

Results Parasitaemia associated negatively with haemoglobin level $\left(r_{s}=-0.43 ; p<0.001\right)$. The plasma level of IL-6 was higher in pregnant women than in non-pregnant women $(p=0.05)$. Regarding parasitaemia, plasma level of IL-27 was significantly higher in non-infected than in infected women $(p=0.028)$ while that of IL-6 was significantly higher in infected women $(p<0.0001)$. Moreover, parasitaemia correlated negatively with the plasma level of IL-27 $(p=0.034)$ and positively with that of IL-6 $(\mathrm{p}<0.0001)$. In addition, level of IL-6 was significantly higher in anaemia-positive than in anaemia-negative women $(p=0.028)$. On the other hand, level of IL-27 negatively associated with the parity $(p=0.022)$ and gestation age $(\mathrm{p}=0.014)$.

Conclusion These results show that in pregnant women, $P$. falciparum malaria infection is associated with high plasma level of IL-6 and low level of IL-27, suggesting that IL-27 could have a protective effect against pregnancy-associated malaria while IL-6 seem to be a potential biomarker of the disease.

\section{PO 8290 INFLUENCE OF THE SICKLE CELL TRAIT ON PLASMODIUM FALCIPARUM TRANSMISSION IN ASYMPTOMATIC CHILDREN}

${ }^{1}$ Christelle Ngou Maffo*, ${ }^{3}$ Antoine Berry, ${ }^{4}$ Isabelle Morlais, ${ }^{1}$ Lionnel B Feufack Donfack, ${ }^{1}$ Albert Bayibeki Ngano, ${ }^{2}$ Sandrine E Nsango, ${ }^{1}$ Elangwe Milo Sarah-Matio, 'Lawrence Ayong. 'Malaria Unit, Centre Pasteur du Cameroun, Yaounde, Cameroon; ${ }^{2}$ Faculty of Medecine University of Douala, Yaounde, Cameroon; ${ }^{3}$ Service de ParasitologieMycologie CHU Toulouse/Université Toulouse III, France; ${ }^{4}$ Equipe MIVEGEC-IRD de Montpellier, France

\subsection{6/bmjgh-2019-EDC.69}

Background The sickle cell trait is associated with protection against severe malaria. Recently, it has been shown that the genetic protection conferred by the sickle cell trait has no effect on the transmission of Plasmodium species from humans to vectors. Our study aimed to investigate the putative association between the sickle cell trait AS and the susceptibility to malaria infection of both the human host and the insect vector.

Methods The study was conducted from June to November 2017 among asymptomatic children living in Cameroon. The samples were collected on microscopy slides, Whatman FTA and grade 17 paper for the selection of gametocyte carriers by microscopy, the molecular diagnosis of Plasmodium species, and sickle cell trait (PCR- RFLP), respectively. Infectivity of the mosquito was measured by experimental infections on gametocyte-containing blood from naturally infected carrier. Genetic diversity was measured using microsatellite markers. Results A total of 1557 children were recruited; the prevalence of Plasmodium infection among this group was 58\% and the AS sickle cell trait $20 \%$. No significant difference in the prevalence of $P$. falciparum infection was observed according to the sickle cell trait carriage and this irrespective of the parasite stage $(p>0.05)$. The level of infectivity of the mosquito was higher when feedings were performed on blood from HbAS genotypes compared to HbAA genotype blood, and the difference was even more significant when the blood pellet was resuspended with non-immune $A B$ plasma $(p<0.0001)$. No significant difference was observed in the infection complexity between HbAS and HbAA genotypes ( $\mathrm{p}>0.05)$.

Conclusion Plasmodium infection is not influenced by HbAS genotype regardless of parasite stage; the risk of anopheles infection is higher with blood from gametocyte carriers with sickle cell trait (HbAS). The sickle cell trait does not affect the multiplicity of infection.

\section{PO 8296 REDUCING LOSS TO FOLLOW-UP OF CHILDREN EXPOSED TO HIV IN THE PROVINCES OF MANICA AND SOFALA, CENTER OF MOZAMBIQUE}

Lúcia da Costa Vieira*, Arlete Mahumane. Beira Operational Research Center, Maputo, Mozambique

\subsection{6/bmjgh-2019-EDC.70}

Background Early childhood diagnosis of HIV is a challenge in many developing countries, including Mozambique. Approximately $50 \%$ of exposed children and HIV-positive are lost to follow-up, i.e. during Postpartum Consultation (CPP), at-risk child consultation (CCR) or ART consultation in the country. The objective was to carry out an intervention to reduce the loss to follow-up of children exposed to and positive for HIV in Manica and Sofala provinces.

Methods Intervention study in HIV-positive women and their children in CPP and CCR in six health facilities in 2016. Stepped-wedge design with 3 cohorts for 3 months of two health facilities randomly selected. Interventions included activist allocation, telephone calls to contact the mothers, guide the mothers with exposed child from CPP to CCR, active outreach to missed mothers, and initiation of ART in the CCR for 3 months. Data were collected from the health facilities and study books. Analysis was binomial logistic regression model with mixed effects.

Results Of the aggregated data, PCR + was 7.7\%, and proportion of HIV-positive women in CPP $17.4 \%$. In the control group only $24 \%$ of the mothers had more than 2 visits with 